\title{
Sur l'estimation de la corrosion de l'almasilium (A.S.G.) par divers produits de nettoyage et de désinfection utilisés dans l'industrie laitière
}

\author{
par \\ G. DAUFIN, Anne-Marie ROBLOT \\ et \\ M. SAINCLIVIER \\ Laboratoire de Recherches de Technologie Laitière, \\ I.N.R.A. (Rennes)
}

Le transport du lait ou de la crème depuis la ferme jusqu'à l'usine laitière s'effectue, pour une grande part, dans des bidons en almasilium. Cet alliage d'aluminium, plus léger que l'acier, est préféré à l'aluminium en raison de sa meilleure résistance mécanique. Comme tous les ustensiles ou appareils ayant été au contact plus ou moins prolongé avec le lait, les bidons doivent être nettoyés et désinfectés quotidiennement avec beaucoup de soins pour éviter toute pollution bactérienne ou chimique du lait, lors de l'utilisation suivante. Mais les produits commerciaux, détergents et désinfectants, peuvent exercer sur l'almasilium une action corrosive appréciable (Daufin et Sainclivier, 1967) qui, par suite de la détérioration de l'état de surface, contrarie l'efficacité détergente et désinfectante. En outre, cette action corrosive peut être telle que l'on doive remplacer très rapidement les bidons corrodés.

Ainsi, le contrôle de la corrosivité des produits de nettoyage et de désinfection présente un grand intérêt du point de vue de la qualité des produits fabriqués et de la longévité du matériel.

Les mesures de la corrosion subie par les bidons en service conduisent à des résultats exacts mais présentent deux graves inconvénients : Elles doivent être effectuées pendant une longue période et ne permettent pas de prévoir les risques encourus.

Ces essais dits "en service » ont été utilisés par Flückiger et Heuscher (1965) sur des bidons à lait en service dans les fermes pour juger la tenue à la corrosion de l'almasilium protégé par des 
couches d'oxyde d'épaisseur et de dureté différentes. Lindqvist et Magnusson (1966) ont effectué un travail semblable. Camphausen (1961) a également utilisé ce type d'essais comme méthode de routine pour estimer la résistance à la corrosion de bidons soumis à l'action d'un certain nombre de cycles de nettoyage dans une machine à laver les bidons. C'est de cette manière que sont conçus les essais dits de "longue durée " pour l'agrément des produits de nettoyage par la D.L.G.* en Allemagne Fédérale.

Les essais accélérés de laboratoire donnent des résultats qui permettent de prévoir rapidement la résistance à la corrosion d'un alliage donné, dans des conditions données. Ces résultats pourront être exploités dans la pratique si l'on a su reproduire, pour les essais, les principaux facteurs de corrosion dans le problème étudié. Zühlsdorf (1962) a utilisé des essais d'immersion-émersion pour évaluer l'action corrosive de 25 produits détergents et désinfectants commerciaux vis-à-vis de l'aluminium 99,5. Seuls 15 d'entre eux se sont avérés peu corrosifs.

Dans l'industrie laitière, le nettoyage et la désinfection du matériel, des appareils ou des ustensiles ayant été au contact du lait, sont suivis d'un rinçage puis d'un séchage. Les techniques présidant à ces opérations varient d'un matériel à l'autre. Elles peuvent consister en un trempage, une circulation ou une pulvérisation de solution détergente, désinfectante ou de rinçage et en un séchage à l'air chaud ou à l'air ambiant.

Nous nous sommes proposé, dans cette étude, d'évaluer la corrosion subie par l'almasilium au cours d'essais accélérés de corrosion comportant la répétition du cycle suivant : nettoyage et/ou désinfection, rinçage, séchage, et de déterminer à l'aide de mesures de pertes de poids et d'observations micrographiques, les produits susceptibles d'être utilisés pour le nettoyage ou la désinfection du matériel en almasilium.

\section{DESCRIPTION DES ESSAIS}

Les conditions pratiques de corrosion des bidons en almasilium sont crées par une succession d'immersions et d'émersions dans les différents milieux. Nous avons recherché à reproduire ces conditions en laboratoire en utilisant un appareil automatique dont la description et le fonctionnement ont fait l'objet d'une publication antérieure (Daufin et Sainclivier, 1968).

* Deutsche Landwirtschaft Gesellschaft. 


\section{Alliage utilisé. Préparation des plaques}

La composition chimique de l'almasilium (A.S.G.) utilisé est la suivante :

\begin{tabular}{c|c|c|c|c|c|c}
\hline $\mathrm{Si}$ & $\mathrm{Mg}$ & $\mathrm{Fe}$ & $\mathrm{Cu}$ & $\mathrm{Zn}$ & $\mathrm{Mn}+\mathrm{Cr}$ & Ti et $\mathrm{Zr}$ \\
1,2 & 1 & $\leqslant 0,5$ & $\leqslant 0,1$ & $\leqslant 0,2$ & $\leqslant 0,3$ & $\leqslant 0,2$ \\
\hline
\end{tabular}

Nous découpons des plaques de dimensions $100 \times 50 \times 2 \mathrm{~mm}$ dans une tôle laminée ayant subi le traitement thermique suivant : trempe à $530^{\circ} \mathrm{C}(1 \mathrm{~h})$ suivie d'un revenu à $175^{\circ} \mathrm{C}(8 \mathrm{~h})$. Les deux faces des plaques et les tranches sont polies mécaniquement sous eau au papier abrasif $n^{\circ} 180$ de granulométrie : $80 \mu$ (fig. 1). Lavées

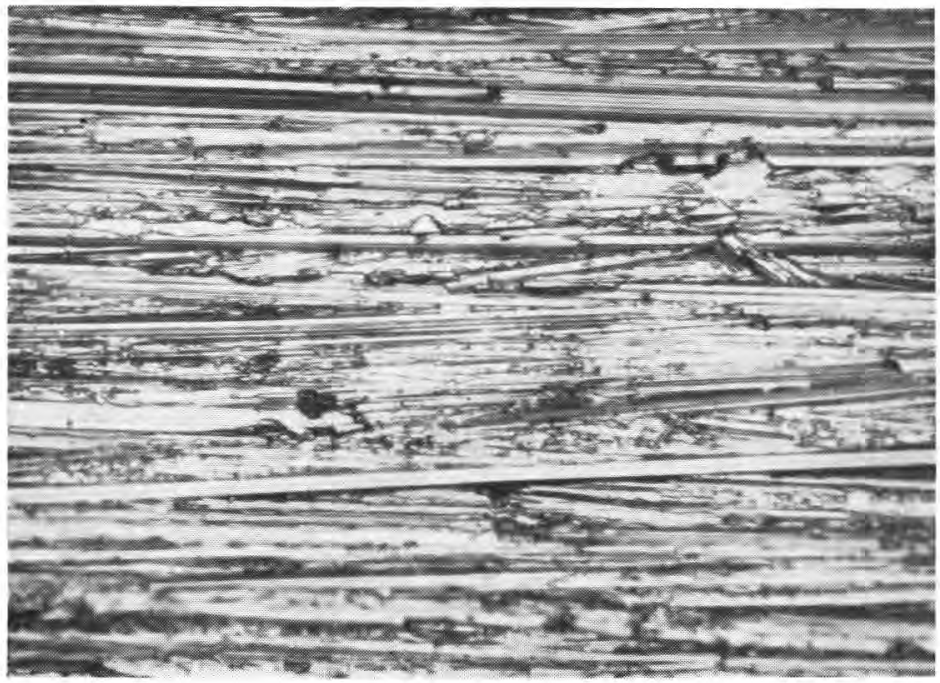

fig. 1

Micrographie de l'état de surface initial obtenu par polissage mécanique sous eau au papier $\mathrm{n}^{\circ} 180$ de granulométrie $80 \mu(\times 200)$

Caractéristiques des rayures : largeur : 10 à $30 \mu$ profondeur : 2 à $3 \mu$

à l'eau, les plaques sont ensuite dégraissées à l'alcool et à l'éther, puis mises en dessiccateur jusqu'à obtention d'un poids constant. Afin d'éviter une possible corrosion à la ligne d'eau, la surface non soumise à l'essai est recouverte d'un vernis délimitant une surface d'épreuve de $25 \mathrm{~cm}^{2}$ sur chaque face. 


\section{Essais accélérés d'immersion-émersion}

Les plaques sont immergées ( $50 \mathrm{~cm}^{2}$ de surface) dans $4,5 \mathrm{I}$ de solution détergente et/ou désinfectante. Elles sont rincées dans 4,51 d'eau de la ville de Rennes ( $8^{\circ}$ hydrotimétrique français) puis séchées à l'air ambiant (environ $20^{\circ} \mathrm{C}$ ). La concentration, la température, l'agitation des solutions, la vitesse de rotation des plaques et les durées de chaque opération sont fixées pour la totalité de l'essai. Signalons que ces conditions sont celles conseillées par les fabricants aux utilisateurs des détergents et désinfectants. En ce qui concerne l'agitation, les conseils ont été interprétés de la manière suivante :

- agitation faible : vitesse de rotation des plaques, $20 \mathrm{t} / \mathrm{mn}$;

- agitation moyenne : vitesse de rotation des plaques, $80 \mathrm{t} / \mathrm{mn}$;

- agitation forte : vitesse de rotation des plaques, $100 \mathrm{t} / \mathrm{mn}$.

L'essai comporte 700 cycles (nettoyage - rinçage - séchage), ce qui correspond sensiblement à deux années d'utilisation pratique du matériel, à raison d'un nettoyage par jour. Il convient de préciser ici que le séchage des plaques, pendant l'essai, dure quelques minutes. Il remplace la longue période d'entreposage du matériel (plusieurs heures) entre deux utilisation successives. Cette substitution permet donc de réaliser des essais accélérés sans perturber les phénomènes observés.

Nous avons mesuré la corrosivité de 7 produits détergents et/ou désinfectants commerciaux utilisés dans les conditions prescrites par les fabricants. A titre indicatif nous donnons dans le tableau 1 la nature des éléments entrant dans la composition des produits essayés. Les conditions expérimentales des essais sont résumées dans le tableau 2.

Nous avons également étudié l'influence de la durée d'action de 2 produits sur l'intensité de corrosion de l'A.S.G. Les conditions expérimentales de ces essais sont résumées dans le tableau $3\left(A_{2}, D_{2}\right)$.

\section{Essais dans les conditions d'immersion prolongée}

Nous avons effectué des essais dans des conditions d'immersion totale sur deux des produits étudiés $\left(A_{3}, D_{3}\right.$, tab. 3$)$ afin de déterminer s'il existait une relation entre les résultats obtenus dans des conditions d'immersion-émersion et ceux obtenus dans des conditions d'immersion totale avec une durée totale de contact métal-produit identique.

\section{Mesure de l'intensité de corrosion}

L'intensité de corrosion est mesurée par la perte de poids des plaques lorsqu'il s'agit d'une corrosion uniforme. Dans les cas de corrosion par piqûres, on note le nombre de piqûres par unité de surface, leur profondeur et leur diamètre moyens. 


\section{Composition des formules}

1) Détergents

Sulfate de soude anhydre

Carbonate de soude anhydre

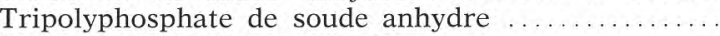

Phosphate disodique anhydre

Hexamétaphosphate de soude

Phosphate disodique cristallise

Phosphate trisodique cristallisé

Métasilicate de soude

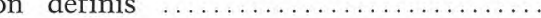

Laurylsulfate de soude

Tensioactifs anioniques

Colorants

Acide phosphorique

\section{2) Désinfectants}

Composé organo-chloré

Chlorure de chaux

Chloramine $\mathrm{T}$

Ammonium quaternaire

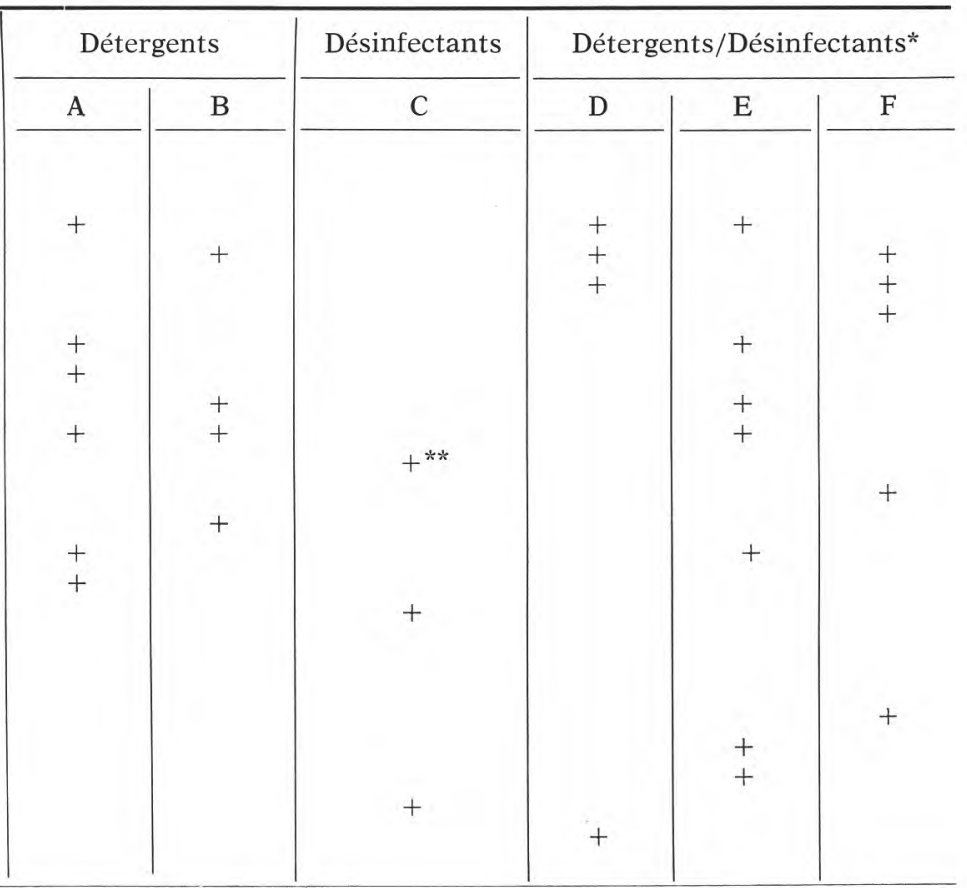

* Nous n'avons pas pu connaître la composition du produit G.

** En dépit de l'existence de «Détergents non définis " dans la formule du produit C, celui-ci est considéré comme un désinfectant et non comme un Détergent/Désinfectant. 
TABLEAU 2. - Description des essais effectués dans les conditions conseillées par les fabricants

\begin{tabular}{|c|c|c|c|c|c|c|c|c|c|c|}
\hline \multirow[t]{2}{*}{ Produit } & \multicolumn{5}{|c|}{ Nettoyage } & \multicolumn{3}{|c|}{ Rinçage à l'eau } & \multicolumn{2}{|c|}{ Séchage à l'air ambiant } \\
\hline & $\mathrm{C}$ & $\mathrm{pH}$ & $\theta$ & $\mathrm{t}$ & Agitation & $\theta$ & $\mathrm{t}$ & Agitation & $\mathrm{t}$ & Agitation \\
\hline A & 8,3 & 10,5 & 50 & 10 & non & 50 & 1 & non & 2 & non \\
\hline B & 5,0 & 12,0 & 45 & 3 & non & 20 & 5 & non & 15 & non \\
\hline $\mathrm{C}$ & $50^{*}$ & 2,7 & 25 & 10 & faible & 20 & 1 & faible & 8 & faible \\
\hline $\mathrm{D}$ & 5,0 & 9,3 & 50 & 3 & moyenne & 50 & 1 & moyenne & 2 & moyenne \\
\hline $\mathrm{E}$ & 10,0 & 7,2 & 50 & 20 & non & 20 & 2 & non & 14 & non \\
\hline $\mathrm{F}$ & 3,3 & 10,2 & 45 & 3 & forte & 20 & 5 & forte & 15 & forte \\
\hline G & 15,0 & 11,4 & 65 & 10 & forte & 20 & 10 & forte & 12 & forte \\
\hline \multicolumn{6}{|c|}{$\begin{array}{l}\mathrm{t}=\text { Durée du nettoyage et/ou de la désinfection en } \mathrm{mn} . \\
{ }^{*}=\text { Concentration exprimée en } \mathrm{ppm} \mathrm{d}^{\prime} \mathrm{I}_{2}\end{array}$} & $\begin{array}{l}\text { Cono } \\
\text { Tem }\end{array}$ & $\begin{array}{l}\text { tion } \\
\text { dre }\end{array}$ & oduit en $\mathrm{g}$ & & \\
\hline
\end{tabular}


TABLEAU 3. - Description des essais destinés à l'étude de l'influence de la durée du nettoyage et de la technique d'essai

\begin{tabular}{|c|c|c|c|c|c|c|c|c|c|c|}
\hline \multirow[t]{2}{*}{ Essai } & \multicolumn{5}{|c|}{ Nettoyage } & \multicolumn{3}{|c|}{ Rinçage à l'eau } & \multicolumn{2}{|c|}{ Séchage à l'air ambiant } \\
\hline & $\mathrm{C}$ & $\mathrm{pH}$ & $\theta$ & $\mathrm{t}$ & Agitation & $\theta$ & $\mathrm{t}$ & Agitation & $\mathrm{tmn}$ & Agitation \\
\hline$A_{1}$ & 8,3 & 10,5 & 50 & 10 & non & 50 & 1 & non & 2 & non \\
\hline $\mathrm{A}_{2}$ & 8,3 & 10,5 & 50 & 2 & non & 50 & 1 & non & 2 & non \\
\hline $\mathrm{A}_{3}$ & 8,3 & 10,5 & 50 & 7000 & non & \multicolumn{3}{|c|}{ néant } & \multicolumn{2}{|c|}{ néant } \\
\hline $\mathrm{D}_{1}$ & 5 & 9,3 & 50 & 3 & moyenne & 50 & 1 & moyenne & 2 & moyenne \\
\hline $\mathrm{D}_{2}$ & 5 & 9,3 & 50 & 2 & moyenne & 50 & 1 & moyenne & 2 & moyenne \\
\hline $\mathrm{D}_{3}$ & 5 & 9,3 & 50 & 2100 & moyenne & \multicolumn{3}{|c|}{ néant } & \multicolumn{2}{|c|}{ néant } \\
\hline $\mathrm{C}=$ Conce & en & & & Durée & on $\mathrm{du} p \mathrm{pr}$ & 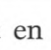 & & empératur & en ${ }^{\circ} \mathrm{C}$. & \\
\hline
\end{tabular}


Dans les essais préliminaires, toutes les plaques étaient pesées (avec leurs produits de corrosion) tous les 100 cycles. La reproductibilité s'étant révélée bonne, dans tous les autres essais, à chaque centaine de cycles une des six plaques est remplacée par une plaque neuve ; cette méthode d'essai est une adaptation de celle de Wachter et Treseder (1947). Elle a permis, dans tous les essais effectués, de vérifier que les pertes de poids, subies par les différentes plaques neuves et mesurées après 100 cycles de corrosion, ne varient pas au cours de l'essai. On peut conclure que la modification des conditions expérimentales (en particulier la concentration des solutions sous l'influence de l'évaporation) est négligeable. Cette méthode d'essai n'a pas permis de mettre en évidence l'influence de la modification de l'état de surface d'une même plaque sur les pertes de poids relatives à un même nombre de cycles de corrosion.

Après élimination du vernis par l'acétone, toutes les plaques, qu'elles soient prélevées après $100,200 \ldots$ ou 700 cycles, sont lavées à l'eau courante puis débarrassées de leurs produits de corrosion éventuels sous l'action des ultra-sons, dans l'eau chaude, dégraissées à l'alcool puis laissées en dessiccateur jusqu'à poids constant. A l'issue de l'essai, on observe toutes les plaques au microscope de métallographie et on note les modifications subies par l'état de surface.

\section{EXPRESSION DES RESULTATS}

Le but essentiel des essais accélérés effectués au laboratoire est de déterminer rapidement si tel produit commercial, ayant satisfait à des essais d'efficacité détergente et/ou désinfectante, est utilisable dans les conditions préconisées par le fabricant sans danger de corrosion pour le matériel. A l'issue de l'essai de corrosion, il est donc nécessaire de connaître la forme de corrosion qui a pris naissance sous l'action du produit envisagé et d'en mesurer l'intensité. Quand on observe une corrosion par piqûres, nous pensons qu'il faut déconseiller l'usage du produit, ainsi que le préconise Mohr (1954). Nous caractérisons cette forme de corrosion par la densité, la profondeur et le diamètre moyens des piqûres ainsi que par la perte de poids bien que celle-ci ne soit pas représentative de la corrosion par piqûres. Dans le cas de la corrosion uniforme la perte de poids permet d'évaluer d'une manière précise l'intensité de la corrosion. Il est donc nécessaire de choisir une unité convenable d'expression de la perte de poids d'une part et une perte de poids maximale acceptable d'autre part.

Mohr (1954) exprime l'intensité de la corrosion en $\mathrm{g} / \mathrm{m}^{2}$ pour $24 \mathrm{~h}$ de contact avec le produit. Ce mode d'expression a, nous semblet-il, un grave inconvénient : Il ne donne pas directement l'intensité de corrosion à laquelle on peut s'attendre dans la pratique. En effet, 24 h de contact avec le produit correspondent, selon les durées 
modifie pas sensiblement l'état de surface afin de ne pas amoindrir l'efficacité du nettoyage ou de la désinfection. En conséquence les résultats que nous donnons comportent la valeur de la perte de poids d'une part et la description de l'état de surface d'autre part.

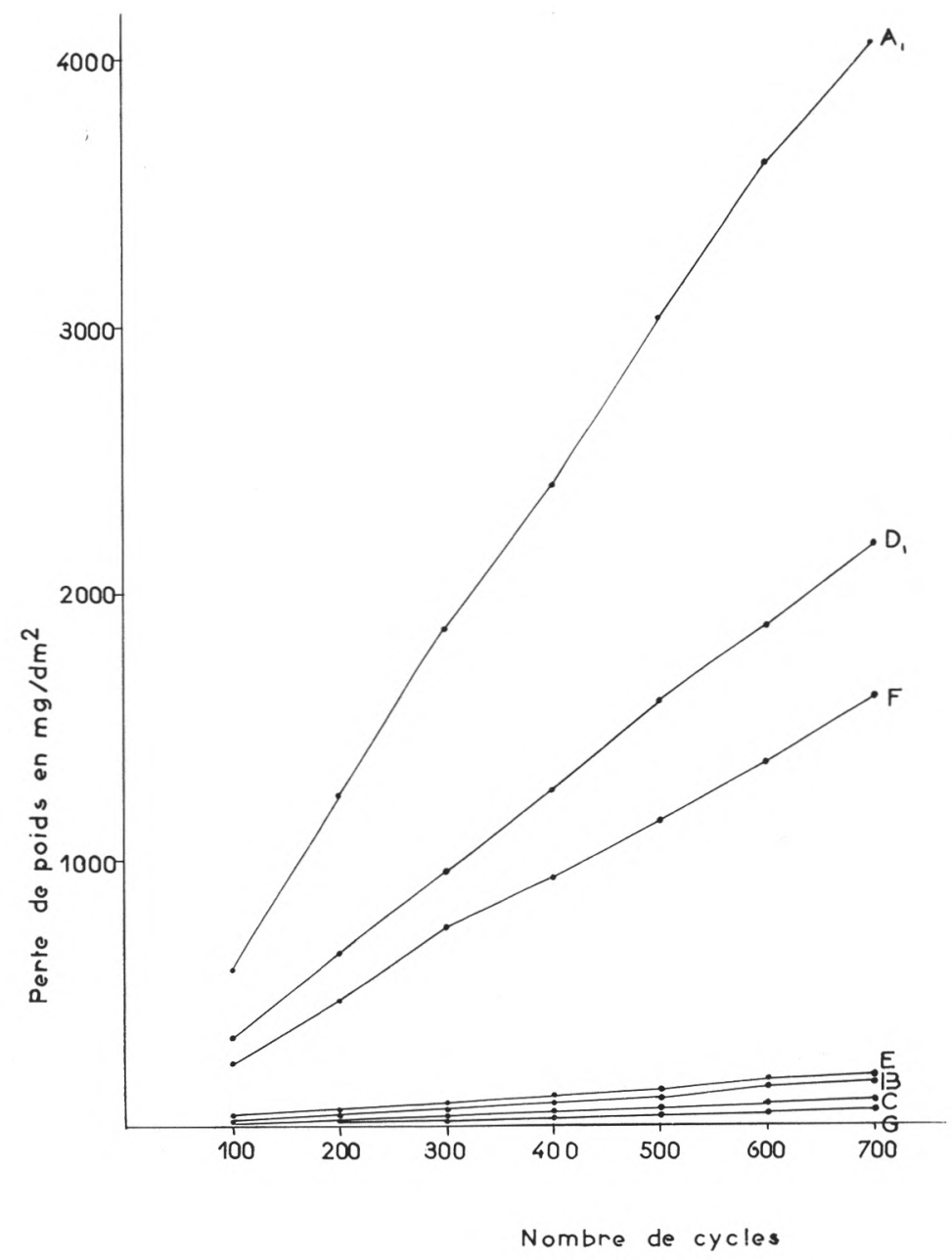

fig. 2

Evolution des pertes de poids en fonction du nombre de cycles : Essais réalisés dans les conditions préconisées par les fabricants 


\section{TABLEAU 4}

Rappel bibliographique des valeurs maximales tolérables correspondant à une bonne résistance à la corrosion de l'almasilium

\begin{tabular}{|c|c|c|c|}
\hline Auteurs & Perte de poids donnée & $\mathrm{mm} / \mathrm{an}^{*}$ & $\begin{array}{c}\text { Perte de poids calculée } \\
\left(\mathrm{mg} / \mathrm{dm}^{2} / \mathrm{an}^{*}\right)\end{array}$ \\
\hline Ritter .......... & $0,7 \mathrm{~g} / \mathrm{m}^{2} / 24 \mathrm{~h}^{*}$ & 0,1 & 2700 \\
\hline Hildebrant & $0,7 \mathrm{~g} / \mathrm{m}^{2} / 24 \mathrm{~h}^{*}$ & 0,1 & 2700 \\
\hline Wiederholt & & 0,1 & 2700 \\
\hline Mohr ... & $20 \mathrm{~g} / \mathrm{m}^{2} / 24 \mathrm{~h}^{* *}$ & 0,004 & 100 \\
\hline Akimov & $0,04 \mathrm{~g} / \mathrm{m}^{2} / \mathrm{h}^{*}$ & 0,1 & 2700 \\
\hline
\end{tabular}

* Durée d'utilisation du matériel.

** Durée de contact avec le produit (équivalent à environ 2 ans d'utilisation du matériel).

d'action des produits, à un plus ou moins grand nombre de cycles de nettoyage et/ou de désinfection, donc de journées d'utilisation réelle. Un reproche semblable peut être fait à l'expression des résultats où l'unité de temps est relative à la durée du cycle de corrosion au laboratoire. Par conséquent nous pensons qu'il faut choisir une unité de temps qui corresponde à la valeur réelle d'utilisation. L'année semble être la durée la plus appropriée puisque nos essais correspondent à deux ans d'utilisation pratique dans le cas d'un nettoyage par jour. Dans la suite de ce texte les résultats de nos essais sont exprimés en $\mathrm{mg} / \mathrm{dm}^{2} /$ année d'utilisation du matériel.

Après avoir adopté une unité d'expression de la perte de poids, il faut choisir une limite maximale pour les pertes de poids afin de déterminer si la résistance à la corrosion de l'A.S.G. est bonne dans les conditions de nos essais. On trouve dans la bibliographie (Akimov 1957, Mohr 1954) un certain nombre de valeurs que nous donnons dans le tableau 4 . Toutes ces valeurs, calculées en $\mathrm{mg} / \mathrm{dm}^{2} /$ an d'utilisation de l'A.S.G., correspondent à une bonne résistance à la corrosion. On constate que Mohr admet une limite $\left(100 \mathrm{mg} / \mathrm{dm}^{2} / \mathrm{an}\right)$ très inférieure à celle des autres auteurs selon lesquels l'almasilium présente une bonne résistance à la corrosion si l'intensité de corrosion ne dépasse par $2700 \mathrm{mg} / \mathrm{dm}^{2} / \mathrm{an}$. Cette limite maximale tolérable permet de juger la résistance à la corrosion de l'almasilium quelles que soient les modalités d'utilisation (durée de contact, températures, nombre de cycles quotidiens, etc.). Signalons que Mohr avoue être en désaccord avec Wiederholt (1945) en ce qui concerne cette limite.

Mais dans l'industrie laitière, il ne suffit pas que la résistance à la corrosion soit bonne, il est aussi nécessaire que la corrosion ne 


\section{RESULTATS}

\section{Essais d'immersion-émersion dans les conditions préconisées par les fabricants}

La figure 2 représente, pour chaque produit, l'évolution de l'intensité de corrosion en fonction du nombre de cycles. Les pertes de poids augmentent régulièrement au cours de tous les essais.

A partir des pertes de poids mesurées à la fin des 700 cycles nous avons calculé, en $\mathrm{mg} / \mathrm{dm}^{2} / \mathrm{an}$ d'utilisation ( 700 cycles correspondant à 700 journées) les pertes de poids moyennes relatives à chaque produit (tab. 5). Ce tableau résume également les observations micrographiques faites sur les plaques ayant subi 700 cycles.

Le produit $A_{1}$ est le plus corrosif des produits essayés (2 220 $\left.\mathrm{mg} / \mathrm{dm}^{2} / \mathrm{an}\right)$. Les produits $\mathrm{D}_{1}$ et $\mathrm{F}$ conduisent à des pertes de poids moins élevées (respectivement 1140 et $860 \mathrm{mg} / \mathrm{dm}^{2} / \mathrm{an}$ ). Dans ces 3 essais l'état de surface est caractérisé par de très nombreuses piqûres qui donnent à la surface l'aspect d'une peau d'orange. A titre d'exemple nous représentons sur la figure 3 la micrographie de la surface à l'issue de l'essai $A_{1}$. Remarquons que le produit $D_{1}$, qui conduit à une perte de poids plus faible que le produit $A_{1}$, entraîne une détérioration plus importante de l'état de surface (densité de piqûres plus élevée, diamètre moyen très supérieur).

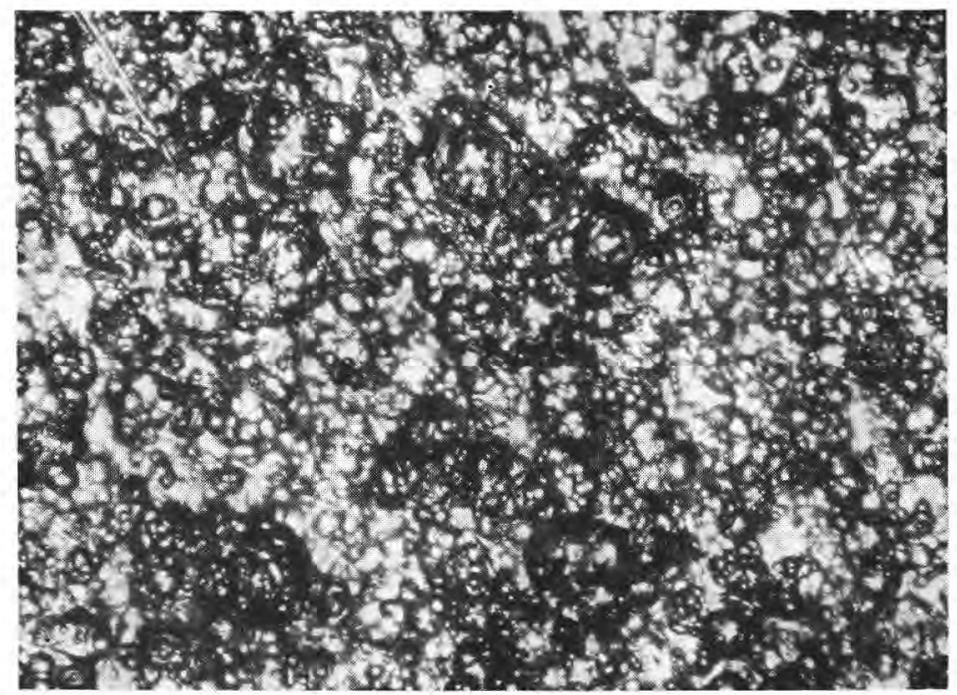

fig. 3

Micrographie de l'état de surface à l'issue de l'essai $A_{1}$ : Exemple d'état de surface "peau d'orange» $(\times 200)$ 
TABLEAU 5. - Résultats des essais réalisés dans les conditions conseillées par les fabricants

\begin{tabular}{|c|c|c|c|c|c|}
\hline \multirow{3}{*}{ Essai } & \multirow{3}{*}{$\begin{array}{l}\text { Pertes de poids moyennes } \\
\left(\mathrm{mg} / \mathrm{dm}^{2} / \text { an d'utilisation }\right)\end{array}$} & \multicolumn{4}{|c|}{ Description de l'état de surface } \\
\hline & & \multirow{2}{*}{ Aspect de la surface } & \multicolumn{3}{|c|}{ Piqûres } \\
\hline & & & $\mathrm{N}$ & $\varnothing$ & $\operatorname{Pr}$ \\
\hline $\mathrm{A}_{1}$ & 2220 & Surface peau d'orange & 10 & $100 \mu$ & $30 \mu$ \\
\hline B & 93 & Rayures visibles, surface mâte & 1 & $50 \mu$ & $10 \mu$ \\
\hline $\mathrm{C}$ & 46 & Rayures un peu visibles & 40 & $300 \mu$ & $100 \mu$ \\
\hline $\mathrm{D}_{1}$ & 1140 & Surface peau d'orange & 12 & $200 \mu$ & $20 \mu$ \\
\hline $\mathrm{E}$ & 103 & Rayures très apparentes & 1 & $25 \mu$ & $5 \mu$ \\
\hline $\mathrm{F}$ & 860 & Surface peau d'orange & 60 & $40 \mu$ & $10 \mu$ \\
\hline G & 34 & Rayures visibles & 4 & $250 \mu$ & $25 \mu$ \\
\hline $\mathrm{N}=$ Dens & piqûres au $\mathrm{cm}^{2}$. & \multicolumn{4}{|c|}{$\operatorname{Pr}=$ Profondeur moyenne. } \\
\hline
\end{tabular}


Les pertes de poids mesurées pour les essais B, C, E et G sont faibles. Le produit $\mathrm{C}$ entraîne la corrosion par piqûres la plus caractéristique (fig. 4) : corrosion uniforme très faible ( $46 \mathrm{mg} / \mathrm{dm}^{2} / \mathrm{an}$ ), rayures encore visibles, piqûres nombreuses, profondes $(100 \mu)$ et larges $(300 \mu)$. Le produit $\mathrm{G}$ conduit à une corrosion uniforme faible et une corrosion par piqûres importante mais moins intense que le produit $C$ (densité, profondeur et diamètre moyen des piqûres moins forts).

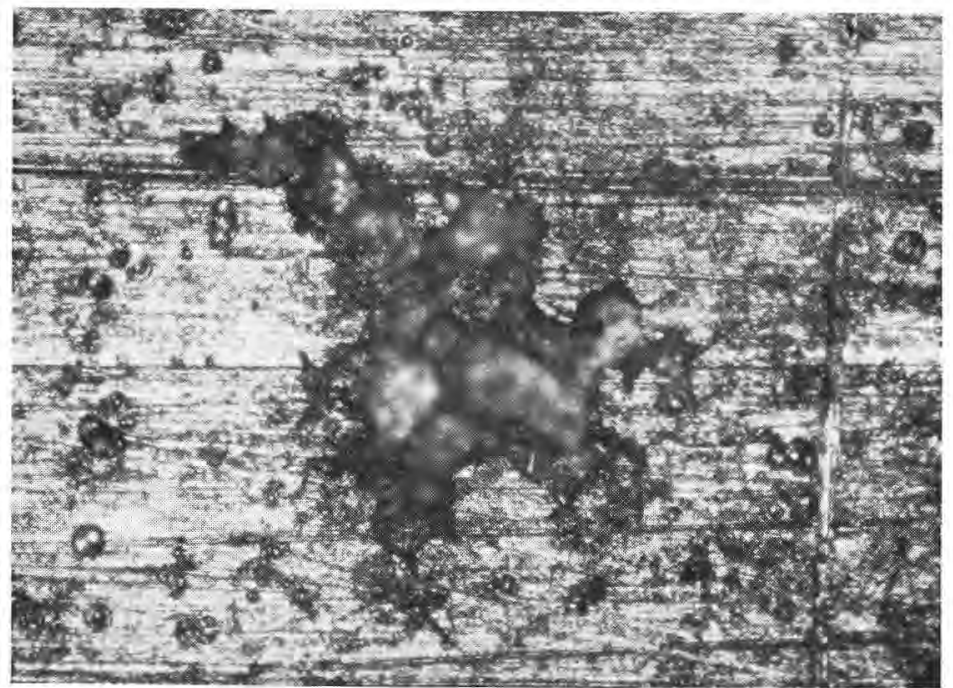

fig. 4

Micrographie de l'état de surface à l'issue de l'essai C : Exemple de corrosion par piqûres caractéristique $(\times 200)$

Enfin les produits B et E sont les moins corrosifs. Les pertes de poids sont faibles (respectivement 93 et $103 \mathrm{mg} / \mathrm{dm}^{2} / \mathrm{an}$ ) et les piqûres peu nombreuses ont de faibles dimensions. A titre d'exemple nous représentons l'état de surface obtenu à l'issue de l'essai B (fig. 5).

\section{Essais d'immersion-émersion dans lesquels la durée de contact diffère de celle préconisée par les fabricants}

L'évolution des pertes de poids mesurées au cours des essais $\mathrm{A}_{2}$ et $\mathrm{D}_{2}$ est indiquée sur la figure 6 et les observations micrographiques faites sur les plaques ayant subi ces essais sont résumées dans le tableau 6.

Pour le produit A, la diminution du temps de contact de $10 \mathrm{mn}$ $\left(\mathrm{A}_{1}\right)$ à $2 \mathrm{mn}\left(\mathrm{A}_{2}\right)$ conduit à une diminution de la perte de poids de 
4 fois environ tandis que la densité et le diamètre moyen de piqûres augmentent.

Pour le produit D, la diminution du temps de contact de $3 \mathrm{mn}$ $\left(D_{1}\right)$ à $2 \mathrm{mn}\left(\mathrm{D}_{2}\right)$ diminue à la fois la valeur de la perte de poids et l'intensité de la corrosion par piqûres.

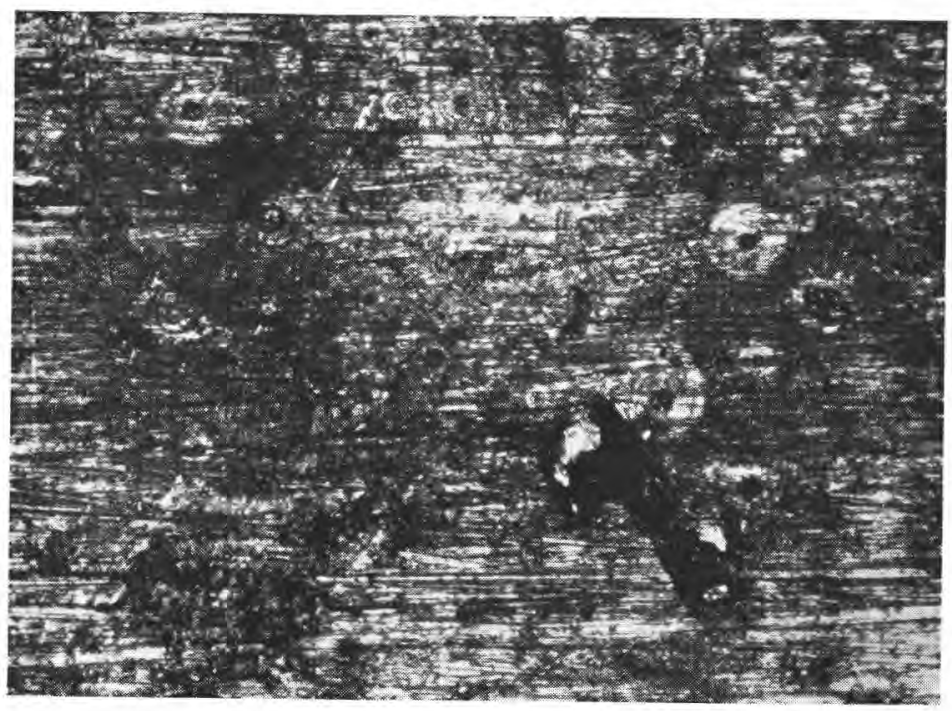

fig. 5

Micrographie de l'état de surface à l'issue de l'essai B : Exemple de corrosion uniforme et de corrosion par piqûres très faibles $(\times 200)$

\section{Essais en immersion prolongée}

Rappelons que la durée d'immersion équivaut à la durée totale de contact entre l'almasilium et le produit au cours de l'essai d'immersion-émersion conforme aux données des fabricants.

La figure 6 et le tableau 6 montrent que pour le produit A, l'essai en immersion prolongée $\left(A_{3}\right)$ conduit à une diminution très sensible de la perte de poids ( 20 fois moins forte que pour $A_{1}$ ) et également à une légère diminution de la corrosion par piqûres. Par contre, pour le produit $\mathrm{D}$, la perte de poids à l'issue de l'essai en immersion prolongée $\left(D_{3}\right)$ est légèrement moins forte que pour l'essai $\mathrm{D}_{1}$ (fig. 6) mais la corrosion par piqûres est beaucoup plus dense et plus intense. 
TABLEAU 6. - Résultats des essais destinés à étudier l'influence du temps de contact et de l'immersion prolongée

\begin{tabular}{|c|c|c|c|c|c|c|}
\hline \multirow{3}{*}{ Essai } & \multirow{3}{*}{ Conditions expérimentales } & \multirow{3}{*}{$\begin{array}{l}\text { Pertes de poids } \\
\text { moyennes à la fin } \\
\text { de l'essai }\left(\mathrm{mg} / \mathrm{dm}^{2}\right)\end{array}$} & \multicolumn{4}{|c|}{ Description de l'état de surface } \\
\hline & & & \multirow{2}{*}{ Aspect de la surface } & \multicolumn{3}{|c|}{ Piqûres } \\
\hline & & & & $\mathrm{N}$ & $\varnothing$ & $\operatorname{Pr}$ \\
\hline $\mathrm{A}_{1}$ & Immersion-émersion $(10 \mathrm{mn})$ & 4160 & Surface peau d'orange & 10 & $100 \mu$ & $30 \mu$ \\
\hline $\mathrm{A}_{2}$ & Immersion-émersion $(2 \mathrm{mn})$ & 1090 & Surface peau d'orange & 12 & $200 \mu$ & $30 \mu$ \\
\hline $\mathrm{A}_{3}$ & Immersion prolongée & 220 & $\begin{array}{l}\text { Rayures légèrement } \\
\text { apparentes }\end{array}$ & 40 & $80 \mu$ & $20 \mu$ \\
\hline $\mathrm{D}_{1}$ & Immersion-émersion $(3 \mathrm{mn})$ & 2190 & Surface peau d'orange & 12 & $200 \mu$ & $20 \mu$ \\
\hline $\mathrm{D}_{2}$ & Immersion-émersion $(2 \mathrm{mn})$ & 1600 & Surface peau d'orange & 16 & $50 \mu$ & $20 \mu$ \\
\hline $\mathrm{D}_{3}$ & Immersion prolongée & 1860 & Surface peau d'orange & 40 & $200 \mu$ & $35 \mu$ \\
\hline
\end{tabular}

$\mathrm{N}=$ Densité de piqûres au $\mathrm{cm}^{2} . \quad \quad \varnothing=$ Diamètre moyen. $\quad \operatorname{Pr}=$ Profondeur moyenne. 


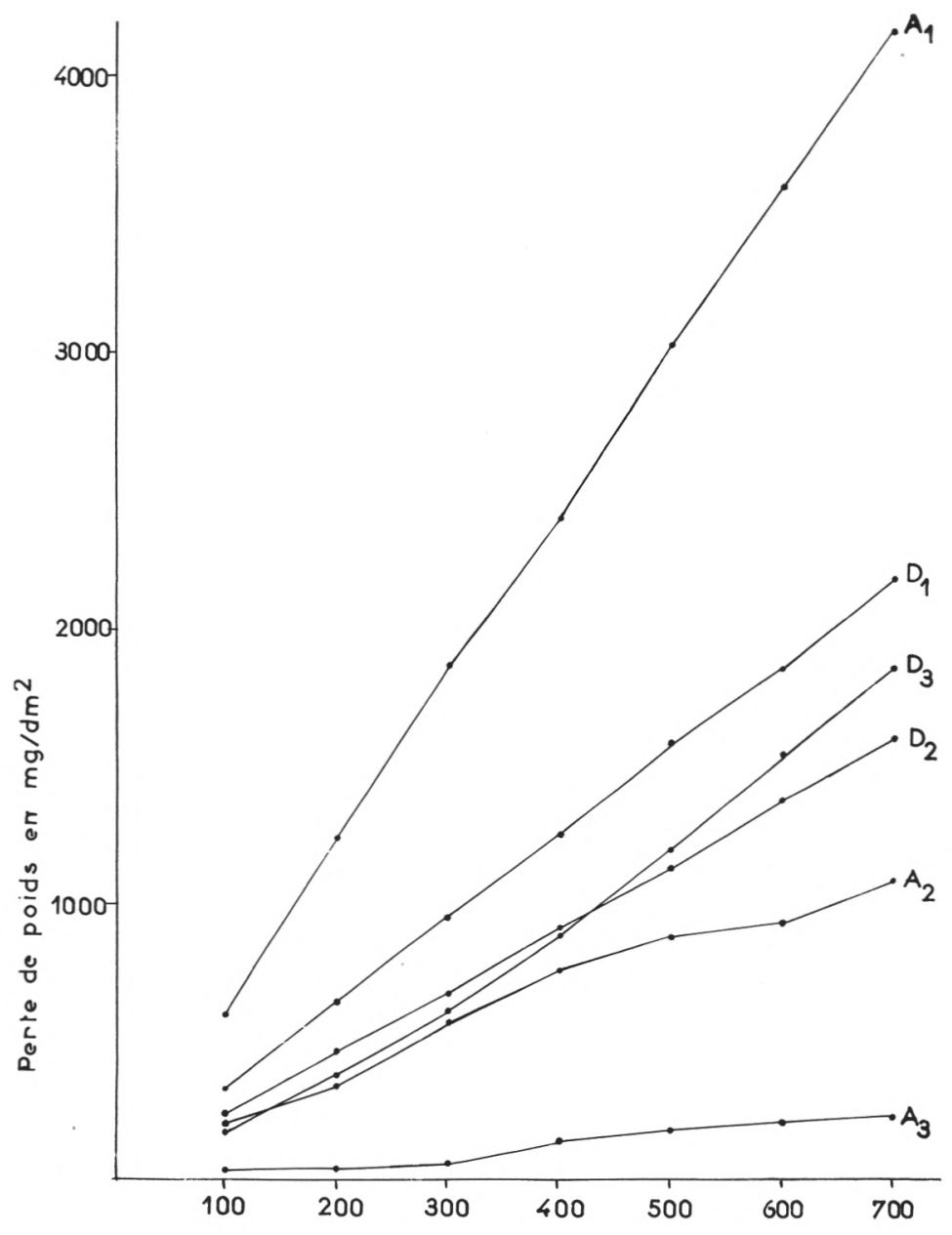

Nombre de cycles

fig. 6

Evolution des pertes de poids au cours des essais en immersion-émersion réalisés avec des durées de contact métal-produit différentes $\left(A_{1}, A_{2}, D_{1}\right.$ $\left.D_{2}\right)$ et au cours des essais en immersion prolongée $\left(A_{3}, D_{3}\right)$ 


\section{DISCUSSION}

\section{Classement des produits}

Il ressort des résultats obtenus (tab. 6) que toutes les pertes de poids mesurées sont inférieures à la perte de poids maximale tolérable que nous nous sommes fixée, soit $2700 \mathrm{mg} / \mathrm{dm}^{2} / \mathrm{an}$. On pourrait en déduire que l'almasilium résiste bien à la corrosion par les produits employés. Mais les observations micrographiques de l'état de surface montrent que l'almasilium présente une corrosion par piqûres à l'issue de tous les essais effectués. Il s'ensuit que tous les produits étudiés sont à déconseiller pour le nettoyage et/ou la désinfection du matériel en almasilium.

Il faut cependant souligner l'importance de la valeur maximale tolérable pour les pertes de poids. En effet, si nous avions choisi $100 \mathrm{mg} / \mathrm{dm}^{2} /$ an, d'après Mohr, nous aurions conclu que l'almasilium ne résistait pas correctement à la corrosion par les produits $\mathrm{A}, \mathrm{D}$, $\mathrm{E}$ et $\mathrm{F}$. Ceci est en désaccord avec nos conclusions puisque ce n'est qu'après les observations micrographiques que nous avons été conduits à déconseiller ces produits.

Les résultats de nos essais ont donc montré que l'état de surface de l'almasilium est fortement détérioré par les cycles de nettoyage et de désinfection étudiés, bien que la résistance à la corrosion uniforme de cet alliage soit bonne dans les conditions expérimentales choisies. Comme aucune relation apparente n'existe entre les pertes de poids et les modifications d'état de surface, nous pensons que la valeur de la perte de poids doit être utilisée dans un premier temps pour éliminer les produits trop corrosifs (perte de poids supérieure à $2700 \mathrm{mg} / \mathrm{dm}^{2} /$ an pour l'almasilium). Ensuite la définition précise de l'état de surface permet d'évaluer le degré de corrosion dans le cas où la perte de poids indique que l'alliage étudié résiste bien à la corrosion uniforme. La mesure des valeurs des surfaces réelles (Thibault et al., 1968) serait un complément très utile de l'observation micrographique des surfaces de même que la mesure de l'aptitude au nettoyage (Daufin et al., 1968).

\section{Influence de la durée de contact}

Les résultats des essais où nous avons modifié la durée d'immersion dans le produit (tab. 6) montrent qu'il n'existe pas de relation apparente entre l'intensité de corrosion et la durée d'action du produit. Il serait donc erroné d'appliquer une règle de proportionnalité pour prévoir les résultats en fonction de la durée de contact, toutes les conditions étant identiques par ailleurs. 


\section{Influence de la technique expérimentale}

Nous avons montré (fig. 6, tab. 6) que les résultats sont totalement différents selon que les essais sont effectués dans des conditions d'immersion-émersion ou d'immersion prolongée. Il est donc impossible de prévoir l'intensité et la forme de corrosion par un produit utilisé dans des conditions d'immersion-émersion à partir des résultats d'un essai simple, réalisé dans des conditions d'immersion prolongée. On peut cependant remarquer que les produits A et $\mathrm{D}$ sont intrinsèquement très corrosifs vis-à-vis de l'almasilium puisque si on exprime l'intensité de corrosion en $\mathrm{mg} / \mathrm{dm}^{2} /$ an d'immersion on trouve respectivement $17400 \mathrm{mg} / \mathrm{dm}^{2} /$ an pour le produit A et $465000 \mathrm{mg} / \mathrm{dm}^{2} /$ an pour le produit D.

\section{CONCLUSION}

Les essais de corrosion réalisés dans des conditions d'immersionémersion montrent que l'almasilium résiste bien à la corrosion uniforme par les 7 produits de nettoyage et/ou de désinfection commerciaux utilisés mais qu'il subit des modifications importantes de l'état de surface (sous forme de piqûres) dans tous les cas. En conséquence les 7 produits utilisés sont à déconseiller pour le nettoyage et la désinfection du matériel laitier en almasilium.

La très grande importance de l'état de surface des alliages utilisés dans l'industrie laitière rend nécessaire l'observation micrographique des surfaces après les essais de corrosion pour compléter et préciser les mesures de la perte de poids. De nouvelles techniques d'évaluation de l'état de surface (mesure directe des surfaces réelles, évaluation de l'aptitude au nettoyage et à la désinfection de ces surfaces) devront être utilisées pour conduire à des conclusions plus objectives.

L'analyse des résultats d'essais de corrosion comportant deux étapes principales (résistance des alliages à la corrosion et modification des états de surfaces), il est nécessaire de normaliser les modes d'expression ainsi que les valeurs maximales tolérables afin que les résultats obtenus puissent être compris par tous les spécialistes des problèmes de corrosion en laiterie.

Les résultats des essais de corrosion réalisés au laboratoire dépendent des conditions expérimentales, en particulier de la durée d'action des produits et de la technique d'essai. Il est donc nécessaire de fixer des conditions expérimentales aussi proches que possible des conditions pratiques. En particulier, on emploiera un alliage possédant la même composition, ayant subi les mêmes traitements thermiques et possédant le même état de surface que l'alliage utilisé dans l'industrie laitière. Les conditions de contact alliage-milieux corrosifs seront reproduites avec le plus de fidélité possible. De cette manière les résultats obtenus auront une bonne valeur d'indi- 
cation sur les risques de corrosion encourus dans le cas particulier de l'étude envisagée bien que les conditions pratiques (formes du matériel, défauts accidentels de surface, technique de nettoyage, durée d'action et concentration des produits, température des solutions, condition d'agitation, etc.) peuvent différer, parfois notablement, des conditions expérimentales précises et reproductibles des essais en laboratoire. En dépit de cette restriction à la valeur des essais de laboratoire, ceux-ci restent particulièrement intéressants pour effectuer des études comparatives entre différents alliages, différents états de surface, différents produits ou différentes conditions de nettoyage ou de désinfection.

\section{Rés um é}

Les auteurs ont effectué des essais de corrosion de l'almasilium (A.S.G.) dans des conditions d'immersion-émersion successives et dans des conditions d'immersion prolongée en utilisant divers produits détergents et/ou désinfectants commerciaux.

Les résultats des essais ont montré que tous les produits sont à déconseiller pour le nettoyage et/ou la désinfection du matériel en almasilium. L'interprétation des résultats nécessite le choix d'une perte de poids maximale acceptable, la mesure des pertes de poids expérimentales et l'évaluation qualitative et quantitative des modifications de l'état de surface.

Il n'existe pas de relation apparente entre les pertes de poids et les modifications d'état de surface ni de règle de proportionnalité entre la durée de contact métal-produit et l'intensité de corrosion d'autre part.

Enfin, les essais réalisés dans des conditions d'immersion-émersion successives et ceux réalisés dans des conditions d'immersion prolongée conduisent à des résultats totalement différents.

\section{S u m m a ry}

The authors tested almasilium (A.S.G.) relating to its corrosion resistance in the so-called conditions of alternate immersions and emersions and of long immersions, by using varied commercial detergent and/or desinfectant products.

The results of the tests showed each product is not to be used in cleaning and/or desinfection. The interpretation of results requires the choice of the highest weight-loss which may be acceptable, the measuring of experimental weight-losses and the qualitative and quantitative evaluation of the modifications of the surface-condition. 
There is neither conspicuous connection between the weightlosses and the modifications of the surface condition nor proportionality-law between the duration of contact metal-product and the corrosion intensity.

Finally, the tests which were achieved in the above-mentionned conditions (that is in alternate immersion and emersions and long immersions) show that the results are entirely different.

\section{Remerciements}

Nous tenons à remercier bien vivement $M$. le Professeur TALBOT, Directeur-Adjoint de l'Ecole Nationale Supérieure de Chimie de Paris, administrateur du Centre Français de la Corrosion, pour les conseils qu'il a bien voulu nous donner pour mener à bien cette publication.

Reçu pour publication le 10 février 1970.

\section{Bibliographie}

- Aкimov (G. V.) (1957). - La corrosion des métaux. 350, Dunod, Paris.

- Camphausen (H.) (1961). - Molk. u. Käs. Ztg., 12, 774-777, 836-837 et 907-910.

- Daufin (G.), Barbier (N.), Kerherve (L.), Roblot (A. M.), Sainclivier (M.) (1968). $-5^{\text {me }}$ Congrès international de la détergence, Barcelone.

- Daufin (G.) et Sainclivier (M.) (1967). - Le Lait, 469-470, 613-632.

- Daufin (G.) et Sainclivier (M.) (1968). - Corr., 16, 5-13.

- Flückiger (E.) et Heuscher (E.) (1965). - Schweiz. Milchztg., 91, 3-8.

- Lindovist (H.) et Magnusson (F.) (1966). - 17 $7^{\text {me }}$ Cong. Int. Lait., F, 493-495.

- Mohr (W.) (1954). - Le nettoyage et la désinfection en laiterie. S.E.P., Paris.

- Thibault (S.), Amouroux (J.), Talbot (J.) (1968). - Corr., 16, 4, 172-176.

- Wachter et Treseder (1947). - Chem. Eng. Progr., 43, 6, 315 et suivantes.

- Wiederholt (1945). - Méthodes d'examen de la corrosion. Verlag Chemie, Berlin.

- Zühlsdorf (M.) (1962). - Deut. Milchw., 9, 263-265. 